\title{
EDITORIAL
}

\section{Comunicación científica: Avances en la profesionalización}

\section{Rubén A. ANANIAS Editor-científico}

Junto a la aparición de la presente edición, la revista Maderas-Ciencia y Tecnología, cumple el décimo aniversario de su selección en Scielo-Chile. Este hito del año 2005, marcó notablemente la necesidad de un cambio en la gestión de manuscritos y de profesionalización de la producción editorial de la revista. El primer cambio fue incorporar además de la producción en papel impreso a la publicación online en la colección Scielo. Este avance implicó la incorporación de un profesional dedicado a la producción editorial de la revista para cumplir con los protocolos y requisitos de la tecnología requerida por Scielo. La digitalización de la revista y su vitrina en Scielo favoreció el acceso a autores y lectores de la revista. Resultando que inmediatamente después, la revista fuera seleccionada e incorporada a Redalyc y Scopus. En adición, el flujo de manuscritos sometidos a la revista se incrementó en un 50\% en los dos primeros años en Scielo, la tasa de rechazo también se duplicó y la producción editorial aumento en un $33 \%$, pasando a producir tres números anuales de manera regular y a tiempo con un incremento de $60 \%$ en artículos científicos publicados.

Por otra parte el año 2010, se avanzó en la implementación de una página web vinculada al sistema de gestión en línea de sumisión de manuscritos OJS (www.revistamaderas.cl). Igualmente las redes sociales Facebook y twitter, fueron incorporadas a través de un proyecto financiado por el fondo de publicaciones científicas del programa Información Científica de Conicyt (Ananías y Salvo 2010). Estando Facebook más activo con cerca de 4500 amigos al presente.

La selección por el Science Citation Index Expanded (Thomson Reuters) del año 2008 y a partir de la edición de la revista Maderas-Cienc Tecnol del año 2007, llego resultado de la gestión profesionalizante de la comunicación científica realizada, que tenía impacto en la disciplina de Ciencias de la Madera de la categoría Materials Science: Paper \& Wood.

Los quince años de la revista, celebrado durante el año 2013, mostraron la construcción del trabajo cada vez más profesionalizante en la comunicación científica de la revista, tanto del punto de vista de la gestión de artículos científicos como de la producción editorial para la plataforma Scielo (Ananías 2013). Resultando que en el Journal Citation Report (JCR-2013), la revista Maderas-Cienc Tecnol alcanzo un factor de impacto a 5 años de 0,698 , lo que indica la creciente tendencia en la evolución de la visibilidad de la comunicación científica a través de la revista en la disciplina de Ciencias de la Madera, categoría Materials-Science: Paper \& Wood (Figura 1). 


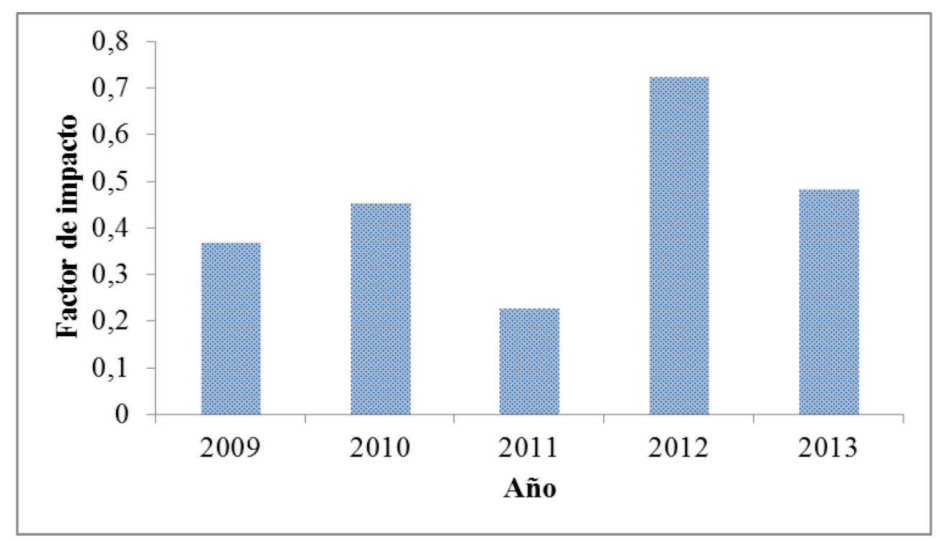

Figura 1: Evolución temporal (5 años), del factor de impacto de la revista Maderas-Cienc Tecnol. (Fuente: Journal Citation Report 2013, Science Edition, Materials Science, Paper \& Wood).

Actualmente se desarrolla la modalidad ahead of print (versión pre-print), para la publicación de los artículos en el sitio Scielo, Chile. Nuevas plataformas, tales como Researchgate, Academia.edu y LinkedIn, se trabaja además en la publicación ahead of print (accepted for authors version) y se exploran, Mendeley y Kudos para mejorar la diseminación de la comunicación científica que se está publicando a través de la revista Maderas-Cienc Tecnol. La profesionalización ha implicado además, la readecuación de las funciones y cargos, creándose los cargos de Editor técnico y Gestor de publicaciones digitales, para apoyar la gestión del Editor cientifico (Rodríguez y Tejada 2013).

Enfin, otro alcance en la comunicación científica de la revista Maderas-Cienc Tecnol, es su creciente participación en la productividad científica de los autores chilenos, tal como registrado por los indicadores de la actividad científica chilena, recogidos en la base de datos Scopus y recientemente revelados (Conicyt-Cis 2014). Dichos indicadores muestran para los últimos 10 años, que en las áreas de Ingeniería Química, Ciencias de los Materiales e Ingeniería, el aporte de la revista es de 4\%, 2\% y $1 \%$ respectivamente, en la comunicación de la ciencia realizada por chilenos.

\section{REFERENCIAS}

Ananías, R.A. 2013. Quince años de la revista Maderas-Ciencia y tecnología: De la infancia a la adolescencia. Maderas-Ciencia y Tecnología 15(3):267-268.

Ananías, R.A.; Salvo, L. 2010. Mejoramiento de la visibilidad de la revista Maderas: Ciencia y tecnología. Poster presentado en Segundo Congreso Internacional de Editores Redalyc, 16-19 noviembre, 2010, Valdivia, Chile.

Conicyt-Cis. 2014. Principales indicadores cienciométricos de la actividad científica chilena 2012. Informe 2014: una mirada a 10 años. Programa de Información Científica. 303p.

Rodríguez, L.; Tejada, C. 2013. El editor técnico: Un perfil necesario para la profesionalización de la edición de revistas científicas en el entorno digital. Anales de documentación 16(2):1-8. 